\title{
Strain-induced spin reorientation of bcc-like iron films grown on $\mathrm{Cu}(001)$
}

\author{
Edna C. Corredor, ${ }^{1,2,3}$ José I. Arnaudas, ${ }^{2,3}$ Miguel Ciria, ${ }^{1,2,{ }^{*}}$ Fabian Lofink, ${ }^{4, \dagger}$ Stefan Rößler, ${ }^{4}$ \\ Robert Frömter, ${ }^{4}$ and Hans Peter Oepen ${ }^{4}$ \\ ${ }^{1}$ Instituto de Ciencia de Materiales de Aragón, Consejo Superior de Investigaciones Científicas, 50009 Zaragoza, Spain \\ ${ }^{2}$ Departamento de Física de la Materia Condensada, Universidad de Zaragoza, 50009 Zaragoza, Spain \\ ${ }^{3}$ Instituto de Nanociencia de Aragón, Universidad de Zaragoza, 50018 Zaragoza, Spain \\ ${ }^{4}$ Institut für Angewandte Physik, Universität Hamburg, Jungiusstr. 11, 20355 Hamburg, Germany \\ (Received 18 June 2014; revised manuscript received 19 October 2014; published 10 November 2014)
}

\begin{abstract}
The in-plane orientation of the magnetization vector $\mathbf{M}$ in bcc-like $\mathrm{Fe}(110)$ films grown on $\mathrm{Cu}(001)$ is determined by means of scanning electron microscopy with polarization analysis. For thicknesses of $2 \mathrm{~nm}$, slightly above the $\mathrm{fcc} / \mathrm{bcc}$ phase transition, it is found that $\mathbf{M}$ is oriented along the $\langle 110\rangle$ directions of the $\mathrm{Cu}(001)$ substrate. Following the Pitsch orientational relationship these correspond to magnetically hard $\langle 1 \overline{1} 1\rangle$ and $\langle 1 \overline{1} 2\rangle$ axes of bulk iron. This finding is in strong contrast to the behavior reported for thicker films (above $3 \mathrm{~nm}$ ) of bcc $\mathrm{Fe} / \mathrm{Cu}(001)$, where the $\langle 100\rangle$ directions of the substrate are preferred. The role of strain in the iron film is discussed, inferring that the presence of a shear strain is mandatory to explain the spin reorientation via the magnetoelastic contribution to the magnetic anisotropy energy.
\end{abstract}

DOI: $10.1103 /$ PhysRevB.90.184410

PACS number(s): 75.30.Gw, 75.50.Bb, 75.70.Cn, 75.60.Ch

\section{INTRODUCTION}

Iron is a widely studied magnetic material that shows a complex behavior either as bulk material [1] or grown as epitaxial film on the $\mathrm{Cu}(001)$ surface [2]. Driven by the magnetic exchange interaction, bulk $\mathrm{Fe}$ crystallizes in a ferromagnetic body-centered cubic (bcc) structure $(\alpha-\mathrm{Fe})$ at room temperature (RT) and exhibits a phase transition to a face-centered cubic $(\mathrm{fcc})$ phase $(\gamma-\mathrm{Fe})$ at $1184 \mathrm{~K}$, which is stable up to $1665 \mathrm{~K}$. The growth of $\mathrm{Fe}$ thin films on $\mathrm{Cu}(100)$ favors the epitaxial growth of the fcc phase in the low-thickness range, due to a small lattice mismatch of $\mathrm{Cu}$ $\left(a_{\mathrm{Cu}}=3.615 \AA\right)$ and fcc $\mathrm{Fe}\left(a_{\mathrm{fcc}-\mathrm{Fe}}=3.58 \AA\right.$, extrapolated value at $\mathrm{RT})$. For $\mathrm{Fe} / \mathrm{Cu}(001)$ grown via thermal evaporation at RT three different regimes have been identified regarding structural and magnetic properties in previous studies [3-13]. A ferromagnetic (FM) phase with perpendicular orientation of the magnetization (regime I) is found for the lowest iron thicknesses, below $\approx 4$ monolayers (MLs). For $t_{\mathrm{Fe}}$ between 5 and 10 MLs an antiferromagnetic (AF) phase covered by a ferromagnetic surface live layer is obtained with a net orientation of magnetization parallel to the normal of the film plane (regime II). A tetragonally distorted fcc (fct) phase is present mainly in regime I while fcc(100) dominates in regime II. Films thicker than 11 MLs show an in-plane magnetization (regime III), which comes along with a martensitic phase transition. The fcc to bec transition exhibits four symmetrically equivalent arrangements (variants) of the bcc $(110)_{b}$ structure on the fcc $(001)_{f}$ substrate with the fcc $\langle 110\rangle_{f}$ and the bcc $\langle 1 \overline{1} 1\rangle_{b}$ directions parallel to each other.

The magnetic anisotropy of the bcc phase has been previously studied, revealing features that indicate a fourfold symmetry with the easy axes along the in-plane $\mathrm{Cu}\langle 100\rangle_{f}$ directions [14-16]. In a ferromagnetic-resonance study it was

\footnotetext{
*Corresponding author: ciria@unizar.es

${ }^{\dagger}$ Present address: Fraunhofer Institute for Silicon Technology, Fraunhoferstr. 1, D-25524 Itzehoe, Germany.
}

shown for films down to $3 \mathrm{~nm}$ thickness that the corresponding anisotropy constants decrease with decreasing film thickness [14]. The extremely small value of the anisotropy constant $K_{1}$ obtained for the lowest thicknesses is in good agreement with the almost isotropic behavior that was found by Scheurer $e t$ al. [15] at $3.6 \mathrm{~nm}$. The resultant anisotropy was recently explained based on the assumption of a strong exchange coupling across the grain boundaries that enforces a homogeneous magnetization orientation [16]. The four different variants that appear with equal probabilities contribute to an averaged first-order anisotropy constant that is obtained by summing up over all orientations utilizing Fe bulk anisotropy constants for the crystallites. As a result the film can be treated as a single-crystalline film (like in the analysis of the data in Ref. [14]), and the magnetic anisotropy reveals a fourfold symmetry and can be described in the conventional form in the coordinate system given by the $\mathrm{Cu}(001)$. An effective anisotropy constant is obtained that is in fairly good agreement with the experimental results for thick films $[14,16]$.

In this paper we present results of an investigation of the magnetic domain pattern by means of scanning electron microscopy with polarization analysis (SEMPA). We address the question for the magnetization orientation within the transition regime from the onset of in-plane magnetization to the almost isotropic behavior found by Scheurer and coworkers [15]. In that thickness range we find an easy axis of magnetization that is close to $\langle 1 \overline{1} 1\rangle_{b}$ in-plane directions, corresponding to the $\langle 110\rangle_{f}$ directions of $\mathrm{Cu}(100)$. To explain the change of easy axes, a magnetoelastic (ME) contribution to the magnetic anisotropy energy is proposed. The shear strain in the (110) plane provides a ME contribution that changes the minimum of the anisotropy energy continuously towards $\langle 1 \overline{1} 1\rangle_{b}$. The shear strain in the Fe (110) plane is calculated utilizing results of previous He scattering experiments [13,17]. Using bulk ME constants, we find that in each crystallite $\mathbf{M}$ deviates by $10^{\circ}$ from the bcc $\mathrm{Fe}\langle 1 \overline{1} 1\rangle_{b}$ directions, which are collinear with the fcc $\mathrm{Cu}\langle 110\rangle_{f}$ axes. Hence, with four different structural variants a fourfold effective magnetic anisotropy has to be expected. 

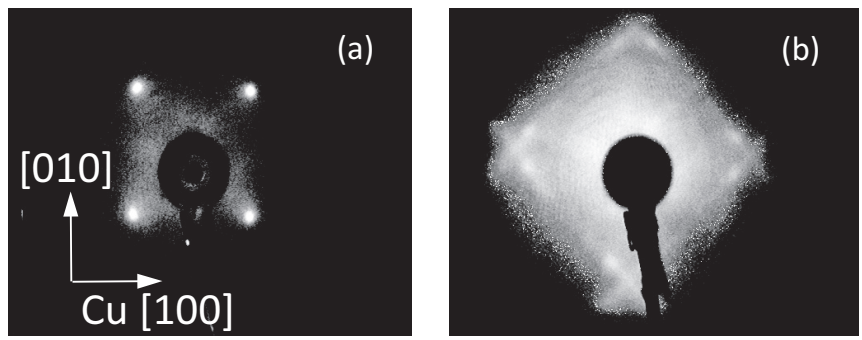

FIG. 1. LEED patterns for (a) clean Cu (100) and (b) 22 ML Fe film. The electron energy is $233 \mathrm{eV}$.

\section{FE FILM GROWTH}

Ultrathin $\mathrm{Fe}$ films were grown on $\mathrm{Cu}(100)$ by e-beam evaporation at RT in ultrahigh vacuum. The base pressure was in the range of $2 \times 10^{-10} \mathrm{mbar}$ and rose to $6 \times 10^{-10} \mathrm{mbar}$ during deposition. Before growth the $\mathrm{Cu}(100)$ substrate was cleaned by cycles of ion milling and annealing. The milling (Ar, $500 \mathrm{eV}, 1 \mu \mathrm{A}$ ) was performed at RT, and subsequent annealing was done at $700{ }^{\circ} \mathrm{C}$ for $20 \mathrm{~min}$. After preparation a sharp $(1 \times 1)$ pattern was observed in low-energy electron diffraction (LEED) for the $\mathrm{Cu}(001)$ substrate; see Fig. 1(a). Fe was evaporated from a high-purity rod at a rate of $0.7 \mathrm{ML} / \mathrm{min}$. An $\mathrm{Fe}$ wedge was fabricated with thickness variation from 0 to 22 MLs by continuous motion of a shutter blade. The LEED pattern from a $22 \mathrm{ML} \mathrm{Fe} \mathrm{film} \mathrm{[Fig.} \mathrm{1(b)]} \mathrm{corresponds} \mathrm{to} \mathrm{the}$ bcc phase. This pattern has been explained as the result of the possible arrangements of the bcc structure on fcc (100) substrates $[6,18,19]$ with the Pitsch orientational relationship, in which rows of nearest-neighbor atoms are matched between $\{011\}_{b}$ bcc and $\{001\}_{f}$ fcc planes [19]. The latter means that the $\langle 1 \overline{1} 1\rangle_{b}$ direction matches the $\langle 110\rangle_{f}$ axis and four different but geomatrically equivalent variants occur for $\mathrm{Fe}$ on $\mathrm{Cu}(100)$.

The domain structure was imaged by means of SEMPA. Our setup allows for measuring the two orthogonal in-plane components of magnetization [20]. This allows for precisely determining the in-plane orientation of $\mathbf{M}$ with an angular resolution below $4^{\circ}$ [21]. The magnetic probing depth of SEMPA is less than five atomic layers [22], and the spatial resolution for magnetic structures is about $15 \mathrm{~nm}$. The growth chamber is directly connected to the SEMPA apparatus, and samples can be transferred under ultra-high-vacuum conditions (base pressure $<5 \times 10^{-11} \mathrm{mbar}$ ).

\section{RESULTS AND DISCUSSION}

Figure 2(a) shows the SEM micrograph of the whole Fe wedge. Interesting features are contrast changes that reveal transition lines running parallel to the shutter edge [two vertical lines in Fig. 2(a)]. A bright region appears on the left-hand side. In the second range the signal decreases gradually until it merges into a thickness regime with uniform low intensity. The second transition line is only vaguely discernible because the signal change is very low [see Fig. 2(a)]. This transition, however, can be observed with much stronger, though inverted, contrast when imaging with backscattered electrons; see micrograph in Fig. 2(b).

The change of brightness in Fig. 2(a) can be attributed to changes of the work function, which determines the secondary
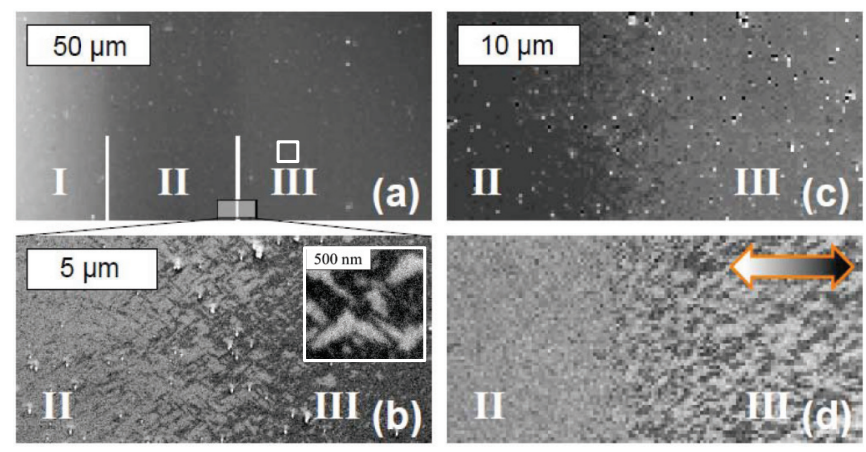

FIG. 2. (Color online) (a) SEM image of the iron wedge. The vertical lines indicate changes of intensity of the secondary electrons. (b) Image taken with backscattered electrons displaying a zoom into the area around the fcc to bcc-like transition. Inset: Image taken at higher magnification showing needle-like fcc domains in a bcc environment. (c) Secondary electron intensity image calculated from the diffraction intensities in the spin detector. Utilizing the same diffraction intensities the polarization image shown in (d) is generated, which implies images (c) and (d) show exactly the same surface region of the wedge.

electron intensity. The material with the lowest work function causes the highest intensity of the secondary electron emission. Values of the work function are $4.6 \mathrm{eV}$ for $\mathrm{Cu}(100)$ [23], $5.5 \mathrm{eV}$ for fcc $\mathrm{Fe}(100)$ [23], and $5.12 \mathrm{eV}$ for bcc $\mathrm{Fe}(110)$ [24]. Hence, the first region in Fig. 2(a) can be appointed to the $\mathrm{Cu}(100)$ surface, and the gradual drop of intensity can be associated with the onset of the iron wedge. Finally the small increase of intensity at the second borderline is due to the phase transition from fcc to bcc-like iron. In the image taken with backscattered electrons the two iron specimens can be clearly resolved forming elongated structures along the $\langle 110\rangle_{f}$ directions. Figures 2(c) and 2(d) display the range where the phase transition appears. Both images were taken simultaneously. At the position of the intensity increase of the secondary electrons the first in-plane magnetized domains appear in the polarization sensitive image; see Fig. 2(d).

The thickness of the iron film, $t_{\mathrm{Fe}}$, has been roughly calibrated utilizing the above-mentioned contrasts under the assumption that during growth the flux of iron and the shutter speed are constant. The wedges were prepared with due care and the thickness determined utilizing the location of the phase transition (fcc to bcc-like) and the onset of the wedge.

In the following we focus our attention on the magnetic structure of bcc-like Fe films. High-resolution SEMPA images of two in-plane polarization components are shown in Figs. 3(a) and 3(b) for an Fe thickness of about 14 MLs (uncertainty $\pm 1 \mathrm{ML}$ ), which corresponds to about $2 \mathrm{~nm}$. The location of the SEMPA images is marked by a square in Fig. 2(a). The high image quality allows for a vectorial summation of the two component images. Figure 3(c) displays the resulting vector representation of the magnetic structure, color coded in accordance to the color wheel. The image shows a distribution of domain sizes ranging from $1 \mu \mathrm{m}$ down to below $300 \mathrm{~nm}$. For an angular analysis of the vectorial image a two-dimensional histogram [Fig. 3(d)] has been calculated. It gives the frequency of all measured orientations of $\mathbf{M}$. The 

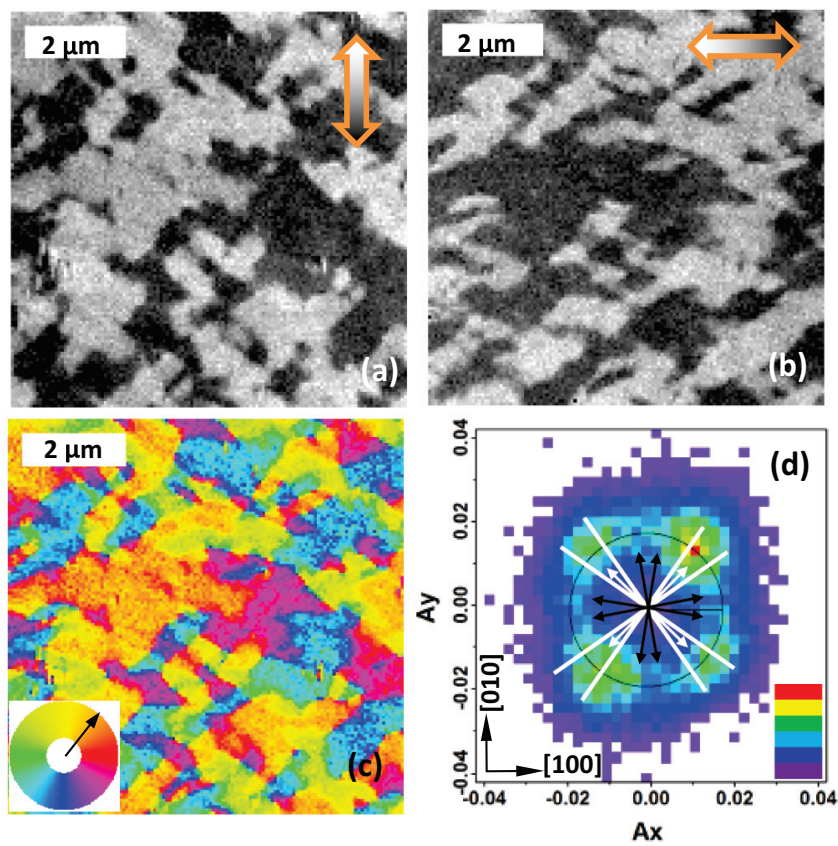

FIG. 3. (Color online) High-resolution SEMPA images showing the magnetic microstructure obtained for two in-plane polarization components, (a) vertical and (b) horizontal. The images are taken simultaneously in the area marked with a square in Fig. 2(a) that corresponds to an iron thickness of $14 \pm 1$ MLs. (c) Magnetic microstructure revealing the distribution of magnetization with in-plane orientation. The image has been calculated by vectorial superpostion of (a) and (b). The magnetization orientation is coded according to the color wheel given as inset. (d) Two-dimensional (2D) histogram of the measured polarization vectors. Here the color represents the frequency of the detected orientation of $\mathrm{M}$, with increasing frequencies changing from blue to red. Ideally, all measured values fall onto one circle as the absolute value of $\mathrm{M}$ should be constant. Due to statistical uncertainty of the measurement a certain scatter of the data around the circle given as a black line is present. The four maxima that can be seen give the magnetization orientations in the domains. Arrows indicate the location of the iron in-plane directions: black arrows for the $\langle 001\rangle_{b}$ and $\langle 1 \overline{1} 0\rangle_{b}$ directions, and white arrows for $\langle 1 \overline{1} 1\rangle_{b}$ and $\langle\overline{1} 12\rangle_{b}$. Thick lines indicate the directions where the minima of the magnetic anisotropy energy are located according to the model described in the text. The polarization sensitive directions of the detector coincide with the [010] and [100] axes of the copper substrate (see arrows in the lower left corner).

maxima represent the orientations of the magnetization in the domains. A fourfold symmetry is found, and the four directions of domain magnetization differ by 90 degrees. These maxima indicate that the magnetization of the domains is along the $\langle 110\rangle_{f}$ directions of the copper single crystal. Taking into account the crystallographic orientation of the $\mathrm{Fe}$ variants [Fig. 1(c)], this corresponds to the Fe $\langle 1 \overline{1} 1\rangle_{b}$ or $\langle\overline{1} 12\rangle_{b}$ directions [these directions are marked with white arrows in Fig. 2(d)].

This finding is in disagreement with previous interpretations and modeling of observed fourfold symmetry [14-16]. Prior to our investigation it has been found for thicker films that the easy axis of magnetization is oriented along the Fe $\langle 100\rangle_{b}$ and $\mathrm{Fe}\langle 1 \overline{1} 0\rangle_{b}$ directions [shown as black arrows in
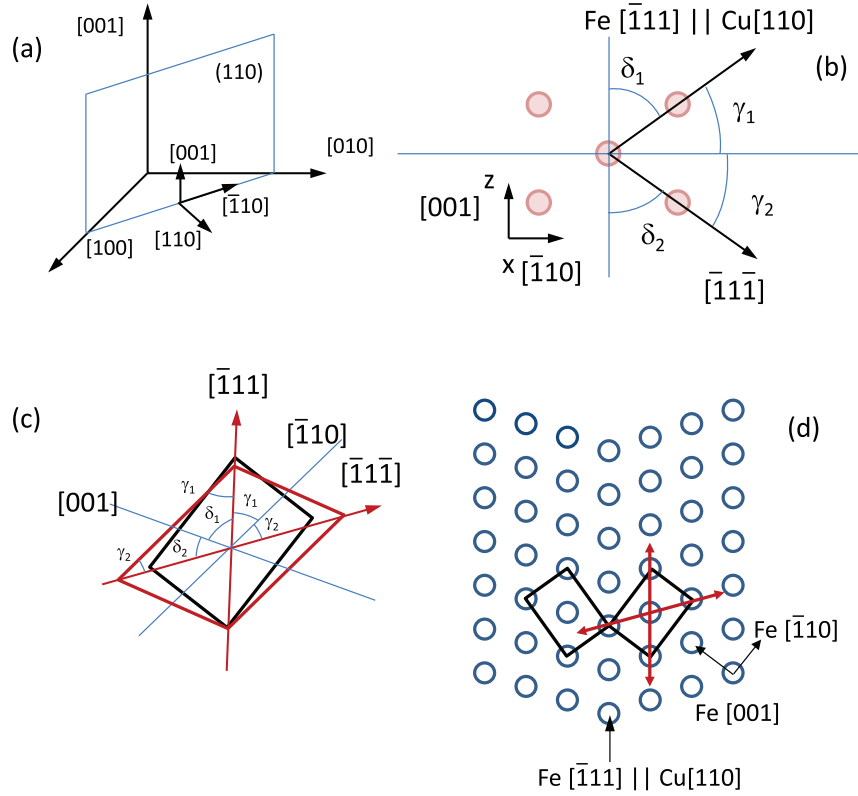

FIG. 4. (Color online) (a) Crystallographic axes and the Fe(110) surface. (b) Cartesian system used in the analysis of the ME energy and location of the Fe atoms on a (110) variant on top of the $\mathrm{Cu}(001)$ surface with the $[\overline{1} 11]_{b}$ direction parallel to the $\mathrm{Cu}[110]_{f}$ direction. (c) Sketch of a shear distortion of the rectangle and (d) sketch of the two variants of the bcc crystallites that share the $[\overline{1} 11]_{b}$ direction. $\delta_{1}$, $\delta_{2}, \gamma_{1}$, and $\gamma_{2}$ indicate the angles between $[\overline{1} 11]_{b}$ and $[\overline{1} 1 \overline{1}]_{b}$ directions with the $[001]_{b}$ and $[\overline{1} 10]_{b}$ axes, respectively.

Fig. 3(d)], which are close to the $\mathrm{Cu}\langle 100\rangle_{f}$ directions. The SEMPA analysis thus indicates that additional contributions to the magnetic anisotropy energy are effective in bcc-like $\mathrm{Fe} / \mathrm{Cu}(001)$ for thicknesses close to the fcc-bcc martensitic transformation.

\section{ANALYSIS}

Well-known and established contributions to the magnetic anisotropy energy $e_{\mathrm{ma}}$ in thin films have their origin in the presence of surfaces and residual strains. Surface terms go with $K_{s} / t_{\mathrm{Fe}}$, with $K_{s}$ being the surface or interface magnetic anisotropy constant. On the other hand, the strains in the film can modify the values of the bulk [25] and surface [26] magnetic anisotropy coefficients. These contributions are significant in $\mathrm{Fe}(110) / \mathrm{W}$ (110) films and explain the switching of $\mathbf{M}$ from the [110] to the [001] in-plane direction as the iron film thickness increases [25,27].

Therefore, the ME energy appears as a natural origin for the new anisotropy observed in the Fe films especially at the thickness of the film studied here. Here 14 MLs is close to the thickness where the martensitic fcc-bcc transformation takes place and residual shear strain is expected to be present in the film.

To describe the ME contribution to the total anisotropy energy $e_{\mathrm{ma}}$, we start considering the ME energy for a cubic symmetry with the coordinate system along orthogonal $\langle 100\rangle$ directions in each crystallite forming the iron film [see Fig. 4(a)] [28,29]. Although the value of the ME coefficients can differ from the bulk values due to strain and surface 
effects, the symmetry of the crystal determines the number of irreducible ME coefficients [30]:

$$
\begin{aligned}
e_{\mathrm{mel}}= & B_{1}\left[\left(\alpha_{z}^{2}-\frac{1}{3}\right)\left(\epsilon_{z z}-\frac{\epsilon_{x x}+\epsilon_{y y}}{2}\right)\right. \\
& \left.+\frac{1}{2}\left(\alpha_{x}^{2}-\alpha_{y}^{2}\right)\left(\epsilon_{x x}-\epsilon_{y y}\right)\right] \\
& +2 B_{2}\left(\alpha_{x} \alpha_{y} \epsilon_{x y}+\alpha_{y} \alpha_{z} \epsilon_{y z}+\alpha_{z} \alpha_{x} \epsilon_{z x}\right) .
\end{aligned}
$$

A rotation of $\pi / 4$ around the [001] direction is more appropriate to describe the $\mathrm{Fe}$ variants, thus for the new coordinate system: $x \|$ [110], $y \|[110]$, and $z \|$ [001] with $\delta_{1}$, $\delta_{2}$ corresponding to angles that form the [001] axis with the [ $\overline{1} 11]$ and $[\overline{1} 1 \overline{1}]$ directions and $\gamma_{1}, \gamma_{2}$ the angles that form the [110] axis with the [ $\overline{1} 11]$ and [ $\overline{1} 1 \overline{1}]$ directions, respectively; see Fig. 4(b). The ME energy reads:

$$
\begin{aligned}
e_{\mathrm{mel}}= & B_{1}\left[\left(\alpha_{z}^{2}-\frac{1}{3}\right)\left(\epsilon_{z z}-\frac{\epsilon_{x x}+\epsilon_{y y}}{2}\right)+2 \alpha_{x} \alpha_{y} \epsilon_{x y}\right] \\
& +B_{2}\left[\frac{1}{2}\left(\alpha_{x}^{2}-\alpha_{y}^{2}\right)\left(\epsilon_{x x}-\epsilon_{y y}\right)+2 \alpha_{x} \alpha_{z} \epsilon_{z x}+2 \alpha_{y} \alpha_{z} \epsilon_{y z}\right] .
\end{aligned}
$$

Any deviation from $90^{\circ}$ of the angle between the [110] and [001] directions [see Fig. 4(b)] can be related to $\epsilon_{y z}$, the shear strain observed in the (110) plane. Note that in the new reference system a change of unit cell in the (110) plane without a change of the angle between the [110] and [100] directions, $\gamma_{1}+\delta_{1}=\gamma_{2}+\delta_{2}=\pi / 2$, is additionally governed by the shear ME coefficient $B_{2}$ because of the presence of $B_{2}\left(\epsilon_{x x}-\epsilon_{y y}\right)$ in Eq. (2). This equation can be simplified by noting that $\mathbf{M}$ is in the film plane so $\alpha_{x}=0, \alpha_{y}=\sin \theta$, and $\alpha_{z}=\cos \theta$. Also, applying the stress boundary conditions at the film surface, $\sigma_{i x}=0$, with $i=x, y, z$, the relationships $\epsilon_{x z}=0, \epsilon_{x y}=0$ and

$$
\epsilon_{x x}=-\frac{\left(c_{11}+c_{12}-2 c_{44}\right) \epsilon_{y y}+2 c_{12} \epsilon_{z z}}{c_{11}+c_{12}+2 c_{44}}
$$

for the strain components utilizing the elastic stiffness constants [31] are obtained. Thus, $e_{\text {mel }}$ becomes

$$
\begin{aligned}
e_{\mathrm{mel}}= & -\left[B_{1}\left(\epsilon_{z z}-\frac{\epsilon_{x x}+\epsilon_{y y}}{2}\right)+B_{2}\left(\frac{\epsilon_{x x}-\epsilon_{y y}}{2}\right)\right] \sin ^{2} \theta \\
& +B_{2} \epsilon_{y z} \sin 2 \theta .
\end{aligned}
$$

The magnetocrystalline anisotropy for the (110) plane is (neglecting terms of order higher than $\sin ^{4} \theta$ )

$$
e_{\mathrm{mc}}=K_{1} \sin ^{2} \theta-\frac{1}{4}\left(3 K_{1}-K_{2}\right) \sin ^{4} \theta .
$$

and the total magnetic anisotropy density energy can be written as

$$
e_{\mathrm{ma}}=\bar{K}_{1} \sin ^{2} \theta-\frac{1}{4}\left(3 K_{1}-K_{2}\right) \sin ^{4} \theta+K_{\mathrm{ME}} \sin 2 \theta
$$

with $K_{\mathrm{ME}}=B_{2} \epsilon_{y z}, \bar{K}_{1}=K_{1}+K_{\mathrm{ME}}^{\prime}$, and

$$
K_{\mathrm{ME}}^{\prime}=-B_{1}\left(\epsilon_{z z}-\frac{\epsilon_{x x}+\epsilon_{y y}}{2}\right)-B_{2}\left(\frac{\epsilon_{x x}-\epsilon_{y y}}{2}\right) .
$$

Therefore, to apply Eq. (6) in the analysis of this in-plane change of easy axis the presence of a significant residual strain in the $\mathrm{Fe}(110)$ layer is mandatory. The $\mathrm{Fe} / \mathrm{Cu}(001)$ is a well-known system because many sophisticated techniques had been used to characterize the crystal structure; here we use the results obtained by grazing-incidence high-energy ion beam techniques $[13,17]$ and I-V LEED [9] to estimate the strain components and the strength of the ME terms of Eq. (4).

The angle between atomic rows has been experimentally measured for bcc-like Fe films grown at $190 \mathrm{~K}$ and RT $[13,17]$. For films in the range of $15 \mathrm{MLs}, \gamma_{1}$ and $\gamma_{2}$ the angles between the $\mathrm{Fe}[\overline{1} 10]_{b}$ axis and the $[\overline{1} 11]_{b}$ and $[\overline{1} 1 \overline{1}]_{b}$ directions, are $36.5^{\circ}$ and $35^{\circ}$, respectively [13]. These values differ from the ideal value $35.26^{\circ}$, and since $\gamma_{1} \neq \gamma_{2}$ the angle between $[\overline{1} 10]_{b}$ and $[001]_{b}$ direction is no longer $90^{\circ}$. This situation causes a shear strain acting on the rectangular cell [see Fig. 4(c)] and allows the determination of $\epsilon_{y z}$. The angles $\delta_{1}$ and $\delta_{2}$ can be evaluated using the law of sines, getting $\delta_{1}=55.7^{\circ}$ and $\delta_{2}=52.8^{\circ}$; therefore $\epsilon_{y z} \approx\left(\pi / 2-\delta_{1}-\gamma_{1}\right) / 2$ can be evaluated obtaining $\epsilon_{y z} \approx-1.9 \times 10^{-2}$.

The knowledge of the angles between atomic rows provides a relationship between the atomic in-plane distances a along the $[100]_{b}$ and $[\overline{1} 10]_{b}$ directions, that is,

$$
\frac{(1 / 2) a_{[100]}}{(1 / 2) a_{[\overline{1} 10]}}=\frac{\epsilon_{z z}+1}{\sqrt{2}\left(\epsilon_{y y}+1\right)}=\frac{\sin \gamma_{1}}{\sin \delta_{1}}=0.72 .
$$

We note that Eqs. (3) and (8) link $\epsilon_{x x}, \epsilon_{y y}$ and $\epsilon_{z z}$, thereby a direct measurement of one the strain components is required to evaluate the term that modifies $K_{1}$; see Eq. (7).

In previous works [2,9] the lattice parameter along the growing $[110]_{b}$ direction has been obtained by means of the I-V LEED technique, showing within the experimental error the match between the values of the film and the bulk lattice parameters. Therefore we assign $\epsilon_{x x} \approx 0$. With this value, and using for stiffness constants of iron $c_{11}=241 \mathrm{GPa}, c_{12}=$ $146 \mathrm{GPa}$, and $c_{44}=112 \mathrm{GPa}$, the in-plane strains can be calculated, obtaining $\epsilon_{y y}=-1.15 \times 10^{-2}$ and $\epsilon_{z z}=0.64 \times 10^{-2}$. Thus, using bulk value for the ME coefficients, $B_{1}=-3.4$ $\mathrm{MPa}$ and $B_{2}=7.6 \mathrm{MPa}$ [32], we obtain $K_{\mathrm{ME}}^{\prime}=-2.2 \mathrm{~kJ} / \mathrm{m}^{3}$. This value is much smaller than $K_{1}=48 \mathrm{~kJ} / \mathrm{m}^{3}$, which implies that $\bar{K}_{1}$ has the same sign as $K_{1}$ and the anisotropy contribution due to the in-plane strain does not change the easy direction with respect to the fully relaxed film. A negative $\bar{K}_{1}$ would cause a switch of the easy axes to $\langle 1 \overline{1} 0\rangle_{b}$ directions. The latter situation, however, cannot be distinguished from a domain pattern with easy axes along $\langle 100\rangle_{b}$ directions since $[100]_{b}$ and $[\overline{1} 10]_{b}$ directions of different variants are parallel to each other. This kind of in-plane easy axis reorientation is observed in $\mathrm{Fe}(110) / \mathrm{W}(110)$ films [27]. In conclusion, the ME contribution to the $\bar{K}_{1}$ term cannot explain the change of easy axes in any case.

The remaining ME contribution in Eq. (6) is proportional to the $\epsilon_{y z}$ shear strain. As the value of this strain is large, $-1.9 \times$ $10^{-2}$, the term $K_{\mathrm{ME}}$ can become the leading contribution in $e_{\mathrm{ma}}$. To highlight the role of this contribution to $e_{\mathrm{ma}}$ we calculate in Fig. 5 the position $\theta_{0}$ of the absolute minima of $e_{\mathrm{ma}}$, as a function of the normalized quantity: $K_{\mathrm{ME}} / K_{1}$, with $K_{\mathrm{ME}}^{\prime}=$ $-2.2 \mathrm{~kJ} / \mathrm{m}^{3}$. It can be seen that $\theta_{0}$ moves away from the $[001]_{b}$ direction to the angular position where the $[\overline{1} 11]_{b}$ and $[\overline{1} 12]_{b}$, or $[\overline{1} 1 \overline{1}]_{b}$ and $[\overline{1} 1 \overline{2}]_{b}$ axes are located, that is, $\pm 35^{\circ}$ and $\pm 54^{\circ}$ depending on the sign of $B_{2} \epsilon_{y z}$. For the case exposed here, $B_{2} \epsilon_{y z}=-144 \mathrm{~kJ} / \mathrm{m}^{3}, \theta_{0} \approx 44^{\circ}$ is obtained, that is, $10^{\circ}$ away 


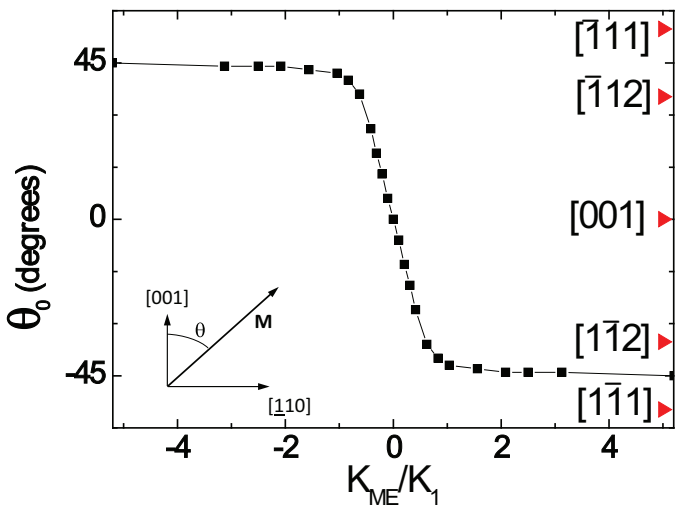

FIG. 5. (Color online) Angular variation of the minimum of $e_{\mathrm{me}}$ as a function of the $K_{\mathrm{ME}} / K_{1}$ calculated with $K_{1}=48 \mathrm{~kJ} / \mathrm{m}^{3}, K_{2}=$ $-10 \mathrm{~kJ} / \mathrm{m}^{3}$, and $K_{1}^{\prime}=-2.2 \mathrm{~kJ} / \mathrm{m}^{3}$.

from the $\mathrm{Fe}[\overline{1} 11]_{b}$ axis (i.e., the $\mathrm{Cu}[110]_{f}$ axis). These easy directions are indicated in Fig. 3(d) as thick white lines in the 2D histogram. They are very close to the orientation of the measured domain magnetization.

This analysis demonstrates that the presence of a minute shear strain can induce a change of easy axes which is in agreement with experimental results for a film thickness of 14 MLs obtained via SEMPA. The model also predicts that the angular deviation from the bulk easy direction is proportional to the shear strain for small $K_{\mathrm{ME}}$ values only $\left(<\sim 0.5 K_{1}\right)$. It is worth mentioning that a change in the sign of $B_{2}$, which has been observed in epitaxial $\mathrm{Fe}(100)$ films in the high-stress regime [32], provides also a satisfactory explanation of the SEMPA data because then $\mathbf{M}$ will be close to the $[\overline{1} 1 \overline{2}]_{b}$ direction, which is perpendicular to the $[\overline{1} 11]_{b}$ and therefore parallel to the $\mathrm{Cu}[1 \overline{1} 0]_{f}$ direction. Nevertheless, using bulk values for $B_{1}$ and $B_{2}$ seems to be reasonable as they have been successfully used to explain the small value of the effective magnetoelastic stress of $\mathrm{Fe} / \mathrm{Cu}(100)$ for iron films thicker than 12 MLs [33].

\section{SUMMARY}

In bcc-like $\mathrm{Fe}(110) / \mathrm{Cu}(100)$ films the magnetic domains are found to be magnetized parallel to the $\mathrm{Cu}\langle 110\rangle_{f}$ directions for thicknesses slightly above the fcc/bcc phase transition. The latter result cannot be explained in the framework of pure magnetocrystalline contribution. Magnetoelastic contributions induced by the shear strain in the $\mathrm{Fe}(110)$ plane provide the strength to overcome the cubic bulk magnetocristalline anisotropy and force the magnetization in directions close to the $\langle 1 \overline{1} 1\rangle_{b}$ direction of each rotational variant. This result reveals the role of strain in such systems and allows for a reinterpretation of previous findings which gave hints of an apparent loss of fourfold anisotropy when the Fe thickness is decreased below 20 MLs. As the thickness increases the strain is relaxed and the system merges into the thick-film limit as predicted by Myagkov et al. [16] Using the strain obtained from available structural data and the bulk ME coefficients, the direction of minimal energy is calculated to be at an angle of $44^{\circ}$ with the $[001]_{b}$ direction and $10^{\circ}$ with the $[\overline{1} 11]_{b}$ and the $\mathrm{Cu}[110]_{f}$ directions. Additional strain components play a minor role and do not alter the symmetry of the magnetic behavior. The fourfold anisotropy is due to the different arrangements of the strained bcc-like variants on the fcc $\mathrm{Cu}$ surface.

\section{ACKNOWLEDGMENTS}

This work has been supported by Spanish MICINN (Grants No. MAT2009-10040 and No. MAT2012-31309) and Gobierno de Aragón (Grants No. E81) and Fondo Social Europeo, as well as by the Deutsche Forschungsgemeinschaft within SFB 668. E.C.C. acknowledges the financial support from Ministerio de Educación, through program Campus de Excelencia Internacional Iberus.
[1] W. Pepperhoff and M. Acet, Constitution and Magnetism of Iron and Its Alloys (Springer, Berlin, Heidelberg, 2001).

[2] M. Wuttig, B. Feldmann, and T. Flores, Surf. Sci. 331-333, Part A, 659 (1995).

[3] C. Liu, E. R. Moog, and S. D. Bader, Phys. Rev. Lett. 60, 2422 (1988).

[4] R. Allenspach and A. Bischof, Phys. Rev. Lett. 69, 3385 (1992).

[5] J. Thomassen, F. May, B. Feldmann, M. Wuttig, and H. Ibach, Phys. Rev. Lett. 69, 3831 (1992).

[6] M. Wuttig, B. Feldmann, J. Thomassen, F. May, H. Zillgen, A. Brodde, H. Hannemann, and H. Neddermeyer, Surf. Sci. 291, 14 (1993).

[7] K. Kalki, D. D. Chambliss, K. E. Johnson, R. J. Wilson, and S. Chiang, Phys. Rev. B 48, 18344 (1993).

[8] D. Li, M. Freitag, J. Pearson, Z. Q. Qiu, and S. D. Bader, Phys. Rev. Lett. 72, 3112 (1994).
[9] P. Schmailzl, K. Schmidt, P. Bayer, R. Döll, and K. Heinz, Surf. Sci. 312, 73 (1994).

[10] S. Müller, P. Bayer, C. Reischl, K. Heinz, B. Feldmann, H. Zillgen, and M. Wuttig, Phys. Rev. Lett. 74, 765 (1995).

[11] J. Giergiel, J. Shen, J. Woltersdorf, A. Kirilyuk, and J. Kirschner, Phys. Rev. B 52, 8528 (1995).

[12] D. E. Fowler and J. V. Barth, Phys. Rev. B 53, 5563 (1996).

[13] T. Bernhard, M. Baron, M. Gruyters, and H. Winter, Phys. Rev. Lett. 95, 087601 (2005).

[14] R. Naik, C. Kota, J. S. Payson, and G. L. Dunifer, Phys. Rev. B 48, 1008 (1993).

[15] F. Scheurer, R. Allenspach, P. Xhonneux, and E. Courtens, Phys. Rev. B 48, 9890 (1993).

[16] V. Myagkov, L. Bykova, and L. Solovyov, J. Magn. Magn. Mater. 322, 1715 (2010).

[17] T. Bernhard, M. Baron, M. Gruyters, and H. Winter, Surf. Sci 600, 1877 (2006). 
[18] K. Heinz, S. Müller, and P. Bayer, Surf. Sci 352-354, 942 (1996).

[19] M. Kato, M. Wada, A. Sato, and T. Mori, Acta Metall. 37, 749 (1989).

[20] R. Frömter, S. Hankemeier, H. P. Oepen, and J. Kirschner, Rev. Sci. Instrum. 82, 033704 (2011)

[21] S. Hankemeier, R. Frömter, N. Mikuszeit, D. Stickler, H. Stillrich, S. Pütter, E. Y. Vedmedenko, and H. P. Oepen, Phys. Rev. Lett. 103, 147204 (2009).

[22] H. P. Oepen and R. Frömter, The Handbook of Magnetism and Advanced Magnetic Materials, Vol. 3 (Wiley, New York, 2007).

[23] P. A. Montano, G. W. Fernando, B. R. Cooper, E. R. Moog, H. M. Naik, S. D. Bader, Y. C. Lee, Y. N. Darici, H. Min, and J. Marcano, Phys. Rev. Lett. 59, 1041 (1987).

[24] R. Fischer, N. Fischer, S. Schuppler, T. Fauster, and F. J. Himpsel, Phys. Rev. B 46, 9691 (1992).
[25] D. Sander, A. Enders, and J. Kirschner, J. Magn. Magn. Mater. 200, 439 (1999).

[26] S. W. Sun and R. C. O'Handley, Phys. Rev. Lett. 66, 2798 (1991).

[27] H. Elmers and U. Gradmann, Appl. Phys. A 51, 255 (1990).

[28] A. Biedermann, M. Schmid, and P. Varga, Phys. Rev. Lett. 86, 464 (2001).

[29] A. Biedermann, R. Tscheliessnig, M. Schmid, and P. Varga, Appl. Phys. A 78, 807 (2004).

[30] J. Rouchy and E. du Tremolet de Lacheisserie, Z. Phys. B 36, 67 (1979).

[31] D. Sander, Rep. Prog. Phys. 62, 809 (1999).

[32] G. Wedler, J. Walz, A. Greuer, and R. Koch, Phys. Rev. B 60, R11313 (1999).

[33] T. Gutjahr-Löser, D. Sander, and J. Kirschner, J. Appl. Phys. 87, 5920 (2000). 